\title{
Equipamentos de pulverização aérea e taxas de aplicação de fungicida na cultura do arroz irrigado ${ }^{1}$
}

\author{
Tânia Bayer ${ }^{2}$, Ivan F. D. Costa ${ }^{3}$, G iuvan Lenz ${ }^{4}$, Carla Zemolin 4 , \\ Leandro N. Marques $\&$ Maurício S. Stefanelo ${ }^{4}$
}

\begin{abstract}
RESU MO
O controle eficiente de doenças na cultura do arroz irrigado depende da seleção correta do equipamento, da taxa de aplicação e da indicação certa do produto químico. O objetivo deste trabalho foi verificar a deposição de gotas na cultura do arroz comparando-se bicos hidráulicos no sistema convencional, eletrostático e atomizador rotativo de disco, em diferentes taxas de aplicação por via aérea; para tanto, foram estudadas a densidade e a penetração de gotas nos estratos médio e inferior, produtividade e rendimento de engenho do arroz. Os tratamentos avaliados foram: bico hidráulico 20 e $30 \mathrm{~L} \mathrm{ha}^{-1}$, eletrostático 5 e $10 \mathrm{~L} \mathrm{ha}^{-1}$ e atomizador rotativo de disco 6,10 e $15 \mathrm{~L} \mathrm{ha}^{-1}$. $\mathrm{N}$ ão foram verificadas diferenças de produtividade e os equipamentos bico hidráulico $20 \mathrm{~L} \mathrm{ha}^{-1} \mathrm{e}$ atomizador rotativo de disco $15 \mathrm{~L} \mathrm{ha}^{-1}$ proporcionaram maior rendimento de engenho. Maiores taxas de aplicação usando-se bicos hidráulicos e atomizador rotativo proporcionaram maior penetração e, consequentemente, também maior densidade de gotas nos dois estratos avaliados.
\end{abstract}

Palavras-chave: bico hidráulico, atomizador de disco, sistema eletrostático, aplicação aérea

\section{Spraying equipment and rates of fungicide application in irrigated rice}

\begin{abstract}
The efficient chemical control in irrigated rice depends on the correct selection of equipment, rates of application and correct statement of the chemical product. The objective of this study was to evaluate the hydraulic nozzle equipment, electrostatic system and rotary-disk atomizer for aerial application of fungicides, relating to penetration and density of droplets with application rates and equipment in the middle and lower strata, in addition to the productivity and hulling yield in irrigated rice. The treatments were: nozzle hydraulic 20 and $30 \mathrm{~L} \mathrm{ha}^{-1}$, electrostatic system 5 and $10 \mathrm{~L} \mathrm{ha-1}$, rotary-disk atomizer 6,10 and $15 \mathrm{~L} \mathrm{ha}^{-1}$. There was no difference in productivity and equipment hydraulic nozzle $20 \mathrm{~L}$ ha ${ }^{-1}$ and rotary-disk atomizer $15 \mathrm{~L} \mathrm{ha}^{-1}$ showed a higher hulling yield. On average, higher rates had higher penetration and density in both the evaluated canopy strata.
\end{abstract}

Key words: hydraulic nozzle, rotary-disk atomizer, electrostatic system, aerial application

\footnotetext{
1 Parte da Dissertação de Mestrado do primeiro autor, apresentada ao PPGAGRO/UFSM

2 DFS/U FPel, campus Capão do Leão, s/n, CP 354, CEP 96010-610, Pelotas, RS. E-mail: tania_bayer@hotmail.com

${ }_{3}^{3}$ DFS/UFSM, Av. Roraima, Bairro Camobi, CEP 97105-900, Santa Maria, RS. Fone: (55)3220-8015, Ramal 34. E-mail: ifdresler@gmail.com

${ }^{4}$ UFPel, Rua Gomes Carneiro, Centro, CEP 96010-610, Pelotas, RS. E-mail: giuvanlenz@gmail.com; carlazemolin@yahoo.com.br; leandronmarques@gmail.com; mstefanelo@hotmail.com
} 


\section{INTRODUÇÃO}

A cultura do arroz irrigado se destaca no sul do País, pela área cultivada e pela produção; entretanto, é acometida por várias doenças passíveis de gerar danos. Dentre essas doenças se destacam patógenos fúngicos causadores de manchas, como a brusone (Pyricularia grisea (Cooke) Sacc), mancha parda (Drechslera oryzae (Breda de Hann) Subr. \& Jain (sin. Bipolaris oryzae), mancha estreita (Cercospora janseana Miyek), escaldadura (Microdochium oryzae), queima das bainhas (Rhizoctonia oryzae Riker \& Gooch) e manchas dos grãos (Phoma sp., Drechslera oryzae, Curvularia lunata, Nigrospora oryzae, Alternaria sp., Fusarium sp.) (Dallagnol et al., 2006).

A aplicação de fungicidas de parte aérea tem-se mostrado medida eficaz para controle dessas doenças (Dallagnol et al., 2006; Celmer et al., 2007). A aceitação desta prática pelos produtores se deve à maior estabilidade na produção, do aumento da qualidade de grãos e ao consistente retorno econômico.

$\mathrm{Na}$ cultura do arroz irrigado a utilização de irrigação por inundação oferece restrições para o uso de equipamentos terrestres para pulverizações, despertando novamente a tecnologia de aplicação aérea com o uso de aeronaves como ferramenta valiosa na agricultura, após uma estagnação na década de 80 , pela baixa eficiência dos serviços prestados (Correa et al., 2004).

A atividade aeroagrícola se mostra como alternativa viável pelo alto rendimento operacional o que permite soluções rápidas em pequenos intervalos de tempo mesmo em grandes extensões de terra; além disso, é possível alcançar resultados satisfatórios com acessível custo econômico, desde que adotados os procedimentos técnicos adequados.

Critérios como equipamentos e volume de aplicação são definidos como essenciais quando se trabalha com aplicações aéreas. A seleção apropriada das pontas determina a quantidade aplicada por área, uniformidade de aplicação, cobertura de gotas e o risco potencial de deriva e, consequentemente, a precisão e segurança na aplicação dos defensivos agrícolas (Womac et al., 1997).

Dentre as pontas utilizadas em aeronaves se destacam bicos hidráulicos, atomizadores rotativos de discos e sistema eletrostático. A pulverização hidráulica é a mais difundida e embasada tecnicamente para aplicações em diferentes situações. Dentre as pontas em bicos hidráulicos, as mais utilizadas são as formadoras de jato plano, que trabalham com pressões menores, geralmente entre 100 e $400 \mathrm{kPa}$, utilizam maiores volumes de calda ( $>100 \mathrm{~L}$ ) e geram gotas relativamente maiores; no entanto, em geral proporcionam pior cobertura do alvo e menor penetração do jato pulverizado no dossel da planta (Cunha et al., 2004).

Nos bicos tradicionais que operam com pressão hidráulica, a formação de gotas é bastante desuniforme e seu tamanho é extremamente desigual dificultando, muitas vezes, uma aplicação eficiente (Cunha et al., 2001); portanto, torna-se necessário avaliar outros equipamentos de aplicação em aeronaves disponíveis no mercado.

Atomizadores rotativos são pontas que giram a alta velocidade, fracionando o líquido em gotas uniformes, com tamanho de gotas pequeno, entre 100 e $200 \mu \mathrm{m}$, possibilitando trabalhar com baixos volumes de calda situando-se entre $10 \mathrm{e}$ $20 \mathrm{~L} \mathrm{ha}^{-1}$; este sistema com uso de atomizadores rotativos é a base da tecnologia conhecida como Sistema BVO $^{\circledR}$ - Baixo Volume Oleoso, desenvolvido pelo CBB em 1998, que trabalha com aplicações de baixos volumes de calda, com adição obrigatória de óleos vegetais ou adjuvantes, que reduzem a evaporação das gotas, permitindo a aplicação de gotas finas com maior eficiência biológica (Monteiro, 2007). Pontas de pulverização com discos rotativos se enquadram dentro da tecnologia Controlled Drop Application (CDA), a qual se caracteriza por aumentar a uniformidade do espectro de gotas, uma vez que elimina gotas muito pequenas, causadoras de deriva e, as muito grandes, que se perdem por escorrimento (Oliveira et al., 2010).

O sistema eletrostático submete as gotas a um potente campo elétrico logo depois de geradas pela energia hidráulica no bico, deixando-as carregadas negativamente para que sejam fortemente atraídas pelas plantas (Schröder \& Loeck, 2006). Hislop (1988) em uma revisão sobre a pulverização eletrostática, afirmou que esta técnica melhora a eficiência da pulverização agrícola através da maior captura e distribuição de gotas em alvos, podendo isto significar redução de $50 \%$ do uso de agrotóxicos sem diminuir a eficácia biológica.

Em alguns países, comoos Estados Unidos e Costa Rica, esta tecnologia é bastante utilizada em pulverizações na cultura do algodão e banana, respectivamente. No Rio Grande do Sul este equipamento foi introduzido em 2001, na cultura do arroz irrigado, para aplicações de fungicidas, herbicidas e inseticidas (Schröder \& Loeck, 2006).

Atualmente, existe uma tendência significativa de se reduzir o volume de calda nas aplicações, técnica justificável pela maior autonomia e a capacidade operacional dos pulverizadores em valores expressivos, podendo ser o principal componente do desempenho operacional em diversas culturas (Román et al., 2009); desta mesma maneira se tenta relacionar o tamanho de gotas à penetração do produto, à uniformidade de distribuição e à efetividade de deposição. A quantificação dessas gotas é facilmente alcançada usando-se o método de Turner \& Huntington (1970) através do uso de papéis sensíveis a água que revelam as gotas que atingem sua superfície (Chaim et al., 1999).

A carência de conhecimento sobre tecnologia de aplicação aérea justificou a condução deste trabalho, que teve como objetivo verificar a deposição de gotas na cultura do arroz irrigado comparando-se bicos hidráulicos no sistema convencional, eletrostático e atomizador rotativo de disco, em diferentes taxas de aplicação por via aérea.

\section{Material e MÉTODOS}

O experimento foi conduzido a campo, em lavoura comercial localizada na Granja Quatro Irmãos, município de Rio Grande, RS, no ano agrícola de 2007/2008. As análises laboratoriais foram realizadas na Clínica Fitossanitária, sediada na Universidade Federal de Santa Maria.

Utilizou-se a cultivar Qualimax 1, semeada no dia 20 de outubro de 2007, através do espaçamento de $0,17 \mathrm{~m}$ entre fileiras 
e densidade de semeadura de 53 sementes por metro linear, o que correspondeu a aproximadamente $85 \mathrm{~kg}$ de semente ha-1. $\mathrm{O}$ experimento foi implantado de acordo com o sistema de plantio convencional e os tratos culturais foram seguidos conforme recomendações técnicas para a cultura do arroz (SOSBAI, 2005).

A área de cada tratamento foi de nove hectares, formada pela passagem de 15 tiros da aeronave Ipanema, modelo 202; dentro de cada talhão foram demarcadas seis áreas de $60 \mathrm{~m}^{2}$ cada uma, constituindo a unidade experimental e, consequentemente as seis repetições de cada tratamento. Em cada talhão, constituído de um tratamento, foi demarcada, próximo às unidades experimentais, uma parcela testemunha, que não recebeu fungicida, também de $60 \mathrm{~m}^{2}$, coberta com lona plástica momentos antes da pulverização; como o trabalho se constituiu de sete tratamentos, uma testemunha por talhão originou sete parcelas/repetição para a avaliação.

O fungicida utilizado no experimento foi a formulação comercial de dois ingredientes ativos, trifloxistrobina + propiconazol, na dose de $0,75 \mathrm{~L} \mathrm{ha}^{-1}$, com concentração de ingrediente ativo de $125 \mathrm{~g}$ de trifloxistrobina $+125 \mathrm{~g}$ de propiconazol; os tratamentos constaram de: 1) Bico hidráulico $20 \mathrm{~L} \mathrm{ha}^{-1}$; 2) Bico hidráulico $30 \mathrm{~L} \mathrm{ha}^{-1}$; 3) Eletrostático $10 \mathrm{~L} \mathrm{ha}^{-1}$; 4) Eletrostático $5 \mathrm{~L} \mathrm{ha}^{-1}$; 5) Atomizador rotativo $15 \mathrm{~L} \mathrm{ha}^{-1}$;6) Atomizador rotativo $10 \mathrm{~L} \mathrm{ha}^{-1}$; e 7) Atomizador rotativo $6 \mathrm{~L} \mathrm{ha}^{-1}$.

Para os tratamentos com uso de atomizadores rotativos de discos adjuvantes foram adicionados à calda, à base de óleo mineral Attach ${ }^{\circledR}$ na dosagem de $0,25 \mathrm{~L} \mathrm{ha}^{-1}$ e de óleo vegetal com características adesivas, espalhante e antievaporante do grupo éster com marca comercial Agróleo ${ }^{\circledR}$ na dosagem de 0,75 $\mathrm{Lha}^{-1}$.

Os tratamentos foram aplicados em 26 de janeiro de 2008 , momento em que as plantas se encontravam na fase de exserção da panícula, segundo escala de Counce et al. (2000). A aplicação foi realizada com a velocidade do vento, que variou entre 15 a $20 \mathrm{~km} \mathrm{~h}^{-1}$, a temperatura entre 24 e $26^{\circ} \mathrm{C}$ e a umidade relativa do ar na faixa de 65 e $72 \%$; todos os tratamentos foram aplicados no tempo de três horas, compreendido entre nove horas da manhã até o meio dia.

Para avaliação de produtividade utilizou-se o peso de grãos, resultante da colheita de $2 \mathrm{~m}^{2}$ em cada unidade experimental; as plantas foram levadas até a Universidade Federal de Santa Maria, onde foram trilhadas, pesadas e determinado o teor de sua umidade; o peso das amostras foi transformado para umidade padrão de $13 \%$ para conversão em $\mathrm{kg} \mathrm{ha}^{-1}$, por último, o rendimento de engenho foi determinado com as mesmas amostras.

Todas as amostras foram submetidas a um conjunto de peneiras para reter qualquer impureza e, logo após, pesados $100 \mathrm{~g}$ para cada repetição; posteriormente, foram beneficiadas em um engenho de prova marca Zaccaria, com o tempo de 1 min, visando à remoção da casca e polimento de grãos, e de 30 s no "trieur" para separar os grãos inteiros dos quebrados.

Os parâmetros considerados na avaliação da qualidade de aplicação foram densidade e penetração de gotas; esses dados foram obtidos a partir da marcação nos cartões hidrossensíveis pelas gotas, informações geradas através da utilização de papéis hidrossensíveis distribuídos sobre o estrato médio e inferior do dossel das plantas; os cartões foram colocados em suportes, compostos por uma estaca e bases metálicas simulando uma folha e distribuídos dentro de cada área de avaliação. Utilizouse a captura de imagem dos cartões com "scanner", em área de no mínimo $1 \mathrm{~cm}^{2}$, com análise da imagem digitalizada através do software Agroscan.

Nas variáveis peso de grãos, produtividade e rendimento de engenho, realizou-se análise da variância e, quando constatada diferença significativa entre os tratamentos, os mesmos foram comparados pelo teste de Duncan a 5\% de probabilidade de erro e as análises foram realizadas com o auxílio do programa estatístico SOC desenvolvido pela EMBRAPA.

Para a análise de equipamentos e taxas de aplicação utilizouse o "software" científico Sisvar ${ }^{\circledR}$, por meio de contrastes ortogonais; analisaram-se contrastes para os diferentes tipos de equipamentos de aplicação (bicos hidráulicos (T1), sistema eletrostático (T2) e atomizadores rotativos de disco (T3)), em primeira análise e, posteriormente, se compararam duas taxas de aplicação utilizadas (alta (TA) e baixa (TB)).

Os contrastes ortogonais analisados foram: contraste 1 (T1)*(T3), contraste 2: (T1)*(T2), contraste 3: (T2)*(T3), e para analisar o efeito das diferentes taxas de aplicação dentro da classificação entre taxas altas e taxas baixas, verificaram-se: os contrastes 1 (T1TA)*(T2TA), o contraste 2: (T1TA)*(T3TA), o contraste 3: (T2TA)*(T3TA), o contraste 4: (T1TB)*(T2TB) e o contraste 5: (T1TB)*(T3TB) e, para efeito de significância, utilizou-se o teste $\mathrm{F}$ admitindo-se $5 \%$ de probabilidade de erro.

\section{RESULTADOS E DISCUSSÃO}

Para os dados de produtividade de grãos (Figura 1), não houve diferença entre os equipamentos usados na pulverização, justificável pela baixa severidade de doenças ocorridas na cultura, a qual não ultrapassou $1 \%$ de área foliar lesionada.

Diferenças na produtividade em soja são encontradas na literatura, com diferentes pontas de pulverização e taxas de aplicação (Cunha et al., 2008); neste ensaio as diferenças foram pequenas em virtude da ocorrência de baixa severidade das doenças.

As flutuações numéricas dos dados são explicadas pelo fato de ser colhida uma pequena área dentro de uma ampla unidade experimental, o que permite interferências de outros fatores, como prováveis desuniformidades da adubação ou da irrigação.

O baixo nível de severidade de doenças implicou também na pequena variação encontrada nos dados de rendimento de engenho (Figura 2), visto que a qualidade dos grãos é parcialmente influenciada pelo fator doenças; a aplicação com bico hidráulico na vazão de $20 \mathrm{~L} \mathrm{ha}^{-1}$ gerou dados superiores de rendimento de engenho, não diferindo do atomizador rotativo de discos com vazão de $15 \mathrm{~L} \mathrm{ha}^{-1}$, indicando que a vazão deve ser definida com base no equipamento utilizado e que, de maneira geral, maiores vazões tendem a apresentar desempenho superior. 


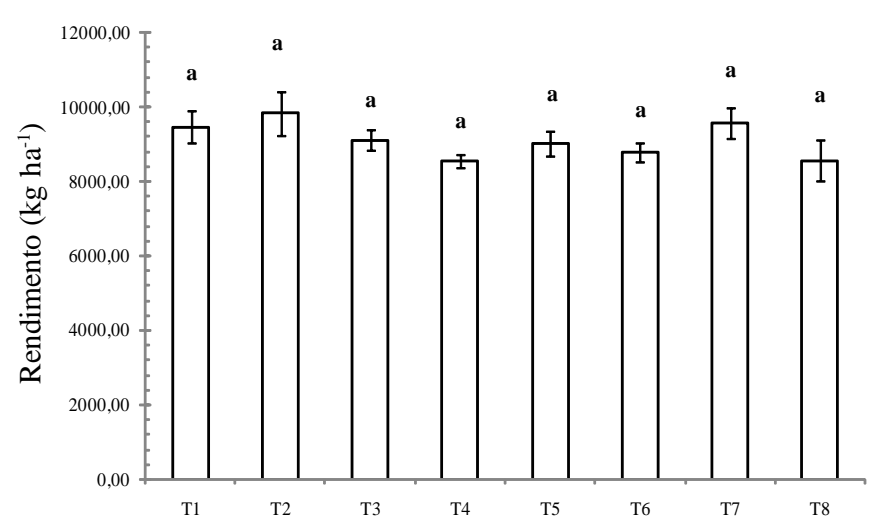

Figura 1. Rendimento de grãos da cultura do arroz irrigado em função da aplicação aérea de fungicidas com diferentes equipamentos e taxas de aplicação. Rio G rande, 2008. Colunas seguidas das mesmas letras não diferem entre si pelo teste de Duncan em nível de $5 \%$ de probabilidade de erro. CV: 10,97\%. T1-Bico hidráulico 30 L ha-1; T2- Bico hidráulico 20 L ha-1; T3- El etrostático $10 \mathrm{~L} \mathrm{ha}^{-1}$; T4- El etrostático $5 \mathrm{~L} \mathrm{ha}^{-1}$; T5- Atomizador rotativo disco $15 \mathrm{~L} \mathrm{ha}^{-1}$; T6- Atomizador rotativo disco $10 \mathrm{~L} \mathrm{ha}^{-1}$; T7-Atomizador rotativo disco $6 \mathrm{~L} \mathrm{ha}^{-1}$; T8- Testemunha

Na Tabela 1 encontram-se os contrastes realizados entre os volumes de aplicações e os equipamentos utilizados nas pulverizações. Os contrastes, comparando-se maiores com menores vazões quanto à penetração no dossel de plantas, se mostraram bastante variáveis, sendo o resultado dependente do equipamento utilizado.

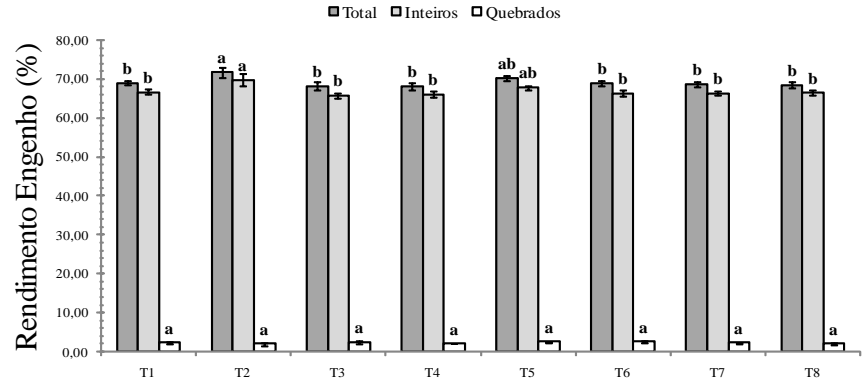

Figura 2. Rendimento de engenho (\%) para amostras de arroz sob uso de diferentes equipamentos e taxas de aplicação aérea de fungicidas na cultura do arroz irrigado. Rio Grande, 2008. Colunas seguidas das mesmas letras dentro de cada categoria não diferem entre si pelo teste de Duncan em nível de $5 \%$ de probabilidade de erro. CV: $3,04 \%$ para peso total, 3,13\% para peso de grãos inteirose $26,62 \%$ para peso de grãos quebrados. T1- Bico Hidráulico 30 L ha-1; T2- Bico hidráulico $20 \mathrm{~L} \mathrm{ha}^{-1}$; T3Eletrostático 10 L ha-1; T4- Eletrostático $5 \mathrm{~L} \mathrm{ha}^{-1}$; T5Atomizador rotativo de disco $15 \mathrm{~L} \mathrm{ha}^{-1}$; T6- Atomizador rotativo de disco $10 \mathrm{~L} \mathrm{ha}^{-1}$; T7- Atomizador rotativo de disco $6 \mathrm{~L} \mathrm{ha}^{-1}$; T8-Testemunha

A penetração das gotas nos estratos médio e inferior foi maior com volumes de calda de 20 e $30 \mathrm{~L} \mathrm{ha}^{-1}$ aplicados com bicos hidráulicos em relação ao sistema eletrostático com menores volumes de calda.

Segundo Murphy et al. (2000), o desvio da trajetória das partículas liberadas pelo processo de aplicação é influenciado

Tabela 1. Contrastes ortogonais entre equipamentos e taxas de aplicação aérea de fungicidas na cultura do arroz irrigado para variáveis penetração e densidade de gotas observadas nos estratos médio e inferior do dossel. Rio G rande, 2009

\begin{tabular}{|c|c|c|c|c|c|c|c|c|}
\hline \multirow{2}{*}{ Taxas } & \multicolumn{4}{|c|}{ Estrato Médio } & \multicolumn{4}{|c|}{ Estrato Inferior } \\
\hline & Média 1 & Média 2 & Fc & $\mathrm{Pr}>\mathrm{Fc} *$ & Média 1 & Média 2 & Fc & Pr> Fc* \\
\hline & \multicolumn{8}{|c|}{ Penetração } \\
\hline BH 30 x Elet 10 & 24,08 & 16,21 & 0,843 & 0,3673 & 9,03 & 6,04 & 0,197 & 0,6611 \\
\hline BH $30 \times$ Atom 15 & 24,08 & 50,35 & 4,674 & 0,0404 & 9,03 & 37,74 & 8,846 & 0,0064 \\
\hline Elet $10 \times$ Atom 15 & 16,21 & 50,35 & 9,486 & 0,0050 & 6,04 & 37,74 & 11,682 & 0,0022 \\
\hline BH 20 x Elet 5 & 25,64 & 23,74 & 0,108 & 0,7453 & 14,43 & 15,02 & 0,079 & 0,7810 \\
\hline \multirow[t]{2}{*}{ BH $20 \times$ Atom 6} & 25,64 & 28,27 & 0,112 & 0,7402 & 14,43 & 14,21 & 0,044 & 0,8348 \\
\hline & \multicolumn{8}{|c|}{ Densidade } \\
\hline BH $30 \times$ Elet 10 & 33,57 & 17,73 & 3,543 & 0,0715 & 12,59 & 6,61 & 1,056 & 0,3140 \\
\hline BH $30 \times$ Atom 15 & 33,57 & 34,50 & 0,012 & 0,9125 & 12,59 & 25,86 & 5,187 & 0,0316 \\
\hline Elet $10 \times$ Atom 15 & 17,73 & 34,50 & 3,973 & 0,0573 & 6,61 & 25,86 & 10,923 & 0,0029 \\
\hline BH 20 x Elet 5 & 24,83 & 11,63 & 2,465 & 0,1290 & 13,98 & 7,36 & 1,292 & 0,2665 \\
\hline \multirow[t]{2}{*}{ BH $20 \times$ Atom 6} & 24,83 & 14,58 & 1,487 & 0,2341 & 13,98 & 7,33 & 1,303 & 0,2644 \\
\hline & \multicolumn{8}{|c|}{ Penetração } \\
\hline $\mathrm{BH} \times$ Atom & 24,72 & 38,08 & 1,502 & 0,2298 & 11,24 & 24,94 & 2,648 & 0,1141 \\
\hline BH x Elet & 24,72 & 18,54 & 0,837 & 0,3675 & 11,24 & 8,81 & 0,018 & 0,8948 \\
\hline Elet $x$ Atom & 18,54 & 38,08 & 6,604 & 0,0154 & 8,81 & 24,94 & 5,657 & 0,0239 \\
\hline $\mathrm{BH} 30 \times \mathrm{BH} 20$ & 24,08 & 25,64 & 0,024 & 0,8792 & 9,03 & 14,43 & 0,170 & 0,6829 \\
\hline Elet $10 \times$ Elet 5 & 16,21 & 23,74 & 0,217 & 0,6448 & 6,04 & 15,04 & 1,351 & 0,2542 \\
\hline \multirow[t]{2}{*}{ Atom $15 \times$ Atom 10,6} & 50,35 & 29,28 & 5,390 & 0,0272 & 37,74 & 15,82 & 8,101 & 0,0079 \\
\hline & \multicolumn{8}{|c|}{ Densidade } \\
\hline $\mathrm{BH} \times$ Atom & 29,20 & 20,70 & 3,484 & 0,0718 & 13,28 & 13,56 & 0,122 & 0,7296 \\
\hline $\mathrm{BH} \times$ Elet & 29,20 & 14,68 & 6,396 & 0,0169 & 13,28 & 6,98 & 2,580 & 0,1187 \\
\hline Elet $x$ Atom & 14,68 & 20,70 & 0,701 & 0,4092 & 6,98 & 13,56 & 3,286 & 0,0799 \\
\hline $\mathrm{BH} 30 \times \mathrm{BH} 20$ & 33,57 & 24,83 & 1,175 & 0,2871 & 33,57 & 13,98 & 0,062 & 0,8046 \\
\hline Elet $10 \times$ Elet 5 & 17,73 & 11,63 & 0,568 & 0,4570 & 6,61 & 7,36 & 0,018 & 0,8934 \\
\hline Atom $15 \times$ Atom 10,6 & 34,50 & 13,80 & 8,698 & 0,0061 & 25,86 & 7,41 & 14,728 & 0,0006 \\
\hline
\end{tabular}


pela velocidade dos ventos, tamanho das gotas, altura de barra e velocidade de deslocamento; neste ensaio as condições de vento se encontravam na faixa de vento fresco, segundo a escala de classificação de Beaufort, adotada pela FAO, podendo ter ocorrido maior perda das pequenas gotas geradas em baixo volume pelo equipamento eletrostático; além disso e devido à ausência de cargas nos cartões hidrossensíveis, eles não apresentam a mesma capacidade de atrair gotas como as plantas, subestimando os valores pela análise.

Nas taxas aplicadas com atomizadores rotativos o volume de $15 \mathrm{~L} \mathrm{ha}^{-1}$ apresentou penetração igual às obtidas com bico hidráulico $30 \mathrm{~L} \mathrm{ha}^{-1}$ e eletrostático $10 \mathrm{~L} \mathrm{ha}^{-1}$, porém a menor taxa de $6 \mathrm{~L} \mathrm{ha}^{-1}$ utilizando atomizador rotativo apresentou penetração superior a uma taxa de $20 \mathrm{~L} \mathrm{ha}^{-1}$ aplicada com bico hidráulico.

Alguns trabalhos da literatura mostram que, quanto menor o tamanho das gotas maior é a penetração de calda para estratos inferiores da cultura (Christofoletti, 1988), mas isto não é regra geral, sendo que no caso do BVO a calda é composta de adjuvantes que condicionam maior vida útil à gota e maiores chances de se alcançar o alvo.

O menor tamanho de gotas remete a baixas taxas de volume aplicadas por hectare, as quais devem ser submetidas a um fracionamento maior da calda; ao se trabalhar na faixa de volume utilizada em aplicações aéreas, que varia de 5 a $30 \mathrm{~L} \mathrm{ha}^{-1}$, verificou-se que a penetração no dossel se comporta diferentemente quando são variados a taxa de aplicação e o equipamento utilizado, sendo que menores taxas aplicadas com sistema eletrostático não apresentaram boa penetração e menores taxas com atomizadores rotativos de disco indicaram maior penetração.

Acredita-se que, quanto menor o tamanho de gotas maior será o seu número aumentando, assim, as possibilidades de ultrapassarem os espaços foliares e chegarem às partes inferiores das plantas. É importante considerar também o tipo de gota formada pelo equipamento e, ainda, quais as características atribuídas à calda, como a adição ou não de adjuvantes, a qual muda totalmente o comportamento da pulverização em diferentes condições climáticas (Cunha \& Carvalho, 2005).

Os contrastes, comparando-se taxas de aplicação com densidade de gotas, mostram dados que seguem a mesma tendência encontrada quanto à penetração no dossel; maiores densidades de gotas foram geradas usando-se maiores volumes de calda por hectare, em ambos os estratos analisados.

No estrato médio os volumes de 20 e $30 \mathrm{~L} \mathrm{ha}^{-1}$ aplicados com bicos hidráulicos apresentaram maior densidade de gotas quando comparados aos menores volumes, 6 e $10 \mathrm{~L} \mathrm{ha}^{-1}$ aplicados com eletrostático, e ao volume de $6 \mathrm{~L} \mathrm{ha}^{-1}$ aplicado com atomizador, porém foi menor a densidade comparado com a aplicação de $15 \mathrm{~L} \mathrm{ha}^{-1}$, com atomizador rotativo.

No estrato inferior bicos hidráulicos $30 \mathrm{~L} \mathrm{ha}^{-1}$ proporcionaram densidade de gotas superior quando comparados ao eletrostático $10 \mathrm{~L} \mathrm{ha}^{-1}$; entretanto, bicos hidráulicos $30 \mathrm{~L} \mathrm{ha}^{-1}$ quando comparados com atomizadores $15 \mathrm{~L} \mathrm{ha}^{-1}$ não se evidenciaram diferenças; maior densidade de gotas foi observada com bicos hidráulicos $20 \mathrm{~L} \mathrm{ha}^{-1}$ quando comparada à do eletrostático $5 \mathrm{~L} \mathrm{ha}^{-1} \mathrm{e}$ à do atomizador $6 \mathrm{~L} \mathrm{ha}^{-1}$.
Oliveira et al. (2010) avaliando o controle de Pseudoplusia includens em condições de laboratório a partir de pulverizações com bico rotativo (17 e $\left.50 \mathrm{~L} \mathrm{ha}^{-1}\right)$ e bico hidráulico $(50$ e $100 \mathrm{~L}$ $\left.\mathrm{ha}^{-1}\right)$ não constataram diferença entre os volumes aplicados; entretanto, isto ocorreu porque o produto atingiu diretamente o alvo, ou seja, em condições de campo talvez isso não ocorra em razão de barreiras físicas, tendendo o melhor desempenho ser alcançado com maior volume, como visto neste trabalho, ao se verificar maior deposição tanto no estrato médio como no inferior.

Segundo Ozeki \& Kunz (1989), a partir de várias experimentações pelo mundo se estabeleceram faixas de densidade de gotas, de acordo com a classe dos produtos, para que se obtenha uma aplicação satisfatória; desta forma, para fungicidas sistêmicos, como é o caso deste ensaio, foi determinada uma faixa de densidade de gotas de 30 a 40 gotas $\mathrm{cm}^{-2}$ para ser considerada uma aplicação satisfatória.

A partir disto pode-se observar que apenas as aplicações de $30 \mathrm{~L} \mathrm{ha}^{-1}$ com bico hidráulico e $15 \mathrm{~L} \mathrm{ha}^{-1}$ aplicado com atomizador geraram dados dentro desta faixa, para o estrato médio, sendo que para o estrato inferior nenhum tratamento atingiu a faixa esperada, constituindo o alvo mais difícil de se atingir (Souza et al., 2007), obtendo-se o melhor resultado com a aplicação de $15 \mathrm{~L} \mathrm{ha}^{-1}$, que ficou próxima de 25 gotas $\mathrm{cm}^{-2}$.

Cunha \& Carvalho (2005) obtiveram maior deposição de gotas no alvo ao usarem maiores volumes de aplicação (20 L $\mathrm{ha}^{-1}$ ); resultado semelhante foi encontrado por Wolf (2000) que, a partir da aplicação aérea de volumes de pulverização de 9 e 29 $\mathrm{L} \mathrm{ha}^{-1}$, encontrou maior cobertura com o maior volume de aplicação.

A redução de volumes de aplicação começou a ser usada em busca de redução de custos e maior rendimento operacional porém volumes de aplicação reduzidos são eficientes quando manuseados corretamente com bicos adequados, de geração e distribuição das gotas, de modo homogêneo e dentro das condições climáticas limites visto que menores volumes implicam em diâmetros menores e, frequentemente, em menor quantidade das gotas depositadas, além de que são mais sujeitas a derivas, causando grandes perdas.

Os contrastes, comparando-se os diferentes equipamentos usados na pulverização, indicam maior penetração de gotas com atomizadores rotativos, fato que está ligado à produção de gotas pequenas e à adição de adjuvantes, que lhes dão maior vida útil, aumentando as chances de atingirem o alvo.

Em geral, alguns adjuvantes tornam a calda mais espessa e com isto, ocorre um aumento no tamanho das gotas, minimizando o arraste causado pelo vento (Cunha \& Carvalho, 2005). Ellis et al. (1997) avaliaram o efeito de adjuvantes na produção de gotas e verificaram que os adjuvantes provocaram alterações significativas no diâmetro e na velocidade das gotas, o que pode estar diretamente relacionado com a redução do risco potencial de deriva; situações como esta confirmam a idéia de que gotas pequenas geradas por atomizadores com adição de adjuvantes à calda, apresentam maior capacidade de penetração no dossel das plantas.

O sistema eletrostático, apesar de gerar gotas pequenas, apresentou menor penetração em relação aos bicos hidráulicos 
e aos atomizadores demonstrando, em um primeiro instante, que a ausência de adjuvantes na calda pode ter sido um dos fatores responsáveis por este desempenho e que isto pode comprometer a qualidade de aplicação quando utilizado este equipamento. A falta de adjuvantes na calda aumenta a sensibilidade das gotas à evaporação e deriva, podendo definir redução do número de gotas depositadas nos cartões, vistas as condições de vento no momento de aplicação $\left(>10 \mathrm{~km} \mathrm{~h}^{-1}\right)$. Cunha \& Carvalho (2005), encontraram densidade de gotas superior depositadas no alvo quando utilizado adjuvante em relação à aplicação apenas com água.

Outros fatores que podem explicar o desempenho inferior em relação aos outros equipamentos é a possibilidade de subestimação dos resultados marcados nos cartões por este equipamento, hipótese também levantada por Schröder \& Loeck (2006), os quais citam que a sensibilidade do sistema Agroscan pode ter comprometido as leituras, por não ter conseguido captar imagens das gotas muito pequenas.

Schröder \& Loeck (2006), também relatam a hipótese das plantas terem atraído as gotas, diminuindo o número de gotas depositadas nos cartões ou podendo haver retenção de gotas no estrato superior, diminuindo a quantidade de gotas interceptadas para estratos inferiores.

Enfim, essas inúmeras hipóteses ajudam a definir que o uso do equipamento eletrostático é muito dependente de condições climáticas e a determinação de sua real penetração e densidade ainda é limitada.

Usando a densidade de gotas como parâmetro para avaliar os equipamentos nos estratos médio e inferior, verificou-se que os bicos hidráulicos proporcionaram densidade de gotas superior à dos atomizadores, porém os atomizadores apresentaram densidade superior ao eletrostático, resultados observados nos dois estratos estudados.

A densidade de gotas tem relação direta com o volume de calda e inversamente proporcional ao diâmetro de gotas; assim, numa situação de aplicação com bicos hidráulicos com maior volume de calda, nota-se tendência à maior densidade de gotas porém com o eletrostático o fator diâmetro de gotas pequeno deveria condicionar um efeito de densidade grande, o que não ocorreu; Cunha et al. (2005) trabalhando com gotas pequenas produzidas por pontas de jato cônico vazio em feijoeiro, esperavam encontrar maior cobertura do alvo, o que também não ocorreu, em condições de campo. Bettini et al. (2007), relataram que condições de ventos em rajadas com velocidade entre 1,2 e 4,8 $\mathrm{km} \mathrm{h}^{-1}$ comprometem a queda das gotas, sendo fator de dificuldade de deposição de calda, principalmente quando é feita aplicação via aérea e com uso de gotas menores que $200 \mu \mathrm{m}$.

Silva et al. (1997), comparando técnicas de pulverização no algodoeiro, concluiram que gotas carregadas eletrostaticamente não foram suficientes para aumentar a capacidade de depósito de gotas $\left(\right.$ gotas $\mathrm{cm}^{-2}$ ) e porcentagem da área coberta em diferentes alturas na face superior das folhas, quando comparada à convencional; estes mesmos autores relatam que isto não ocorreu devido, provavelmente, à baixa tensão de indução de carga na gota, ou seja, a carga não era suficientemente grande para que a gota fosse atraída com eficiência pelas folhas.

A superioridade do equipamento atomizador se relaciona com o uso de adjuvantes à calda, como já mencionado acima, caso em que as gotas formadas são pequenas, porém com características que aumentam as possibilidades de se alcançar estratos inferiores; esses resultados são justificáveis por trabalhos de outros autores, como Cunha \& Carvalho (2005), os quais citam que a densidade de gotas depositadas no alvo utilizando-se adjuvante, foi superior à deposição com água em que, provavelmente, houve redução na evaporação causada pelas condições extremas de umidade.

\section{CONCLUSÕES}

1. Bico hidráulico $20 \mathrm{~L} \mathrm{ha}^{-1}$ e atomizador rotativo disco 15 $\mathrm{L} \mathrm{ha}^{-1}$, promoveram maior rendimento de engenho. Diferença na produtividade de grãos não foi verificada entre os tratamentos.

2. Melhor penetração de gotas no dossel foi obtido com bicos hidráulicos 20 e $30 \mathrm{~L} \mathrm{ha}^{-1}$ e atomizador rotativo de disco $15 \mathrm{Lha}^{-1}$.

3. A adição de óleo vegetal à calda, aplicada por atomizadores, melhora a penetração e aumenta a densidade de gotas.

4. Na avaliação de densidade, comparando-se as taxas de aplicação nos dois estratos, o atomizador rotativo $15 \mathrm{~L} \mathrm{ha}^{-1}$ apresentou, em média, maior número de gotas quando comparado aos demais tratamentos.

5. O atomizador rotativo de disco $15 \mathrm{~L} \mathrm{ha}^{-1}$ apresentou maior penetração no estrato médio e inferior, quando se avaliaram diferentes taxas de aplicação; o mesmo resultado foi encontrado quando se avaliaram equipamentos.

\section{LITERATURA CITADA}

Bettini, P. C.; Miranda, J. E.; Gusmao, L. C. A.; Oliveira Neto, B. A. Avaliação da deposição de gotas em pulverizações terrestres e aérea na cultura do algodoeiro. In: Congresso Brasileiro do Algodão, 6, 2007, Uberlândia. Anais...Uberlândia: EMBRAPA, 2007. CD-Rom

Celmer, A.; Madalosso, M. G.; Debortoli, M. P.; Navarini, L.; Balardin, R. S. Controle químico de doenças foliares na cultura do arroz irrigado. Pesquisa Agropecuária Brasileira, v.42, p.901-904, 2007.

Chaim, A.; Maia, A. H. N.; Pessoa, M. C. P. Y. Estimativa de deposição de agrotóxicos por análise de gotas. Pesquisa Agropecuária Brasileira, v.34, p.963-969, 1999.

Christofoletti, J. C. O uso de bicos de pulverização para aplicações aéreas. Revista Voar, n.61-A, p.22-24, 1988.

Correa, H. G.; Benez, S. H.; Berton, R. S.; Saes, L. A. Depósitos de calda obtidos com a aplicação aérea de defensivos na cultura da banana. Bragantia, v.63, p.121-128, 2004.

Counce, P.; Keisling, T. C.; Mitchell, A. J. A uniform, objective, and adaptive system for expressing rice development. Crop Science, v.40, p.436-443, 2000. 
Cunha, J. P. A. R.; Carvalho, W. P. A. Distribuição volumétrica de aplicações aéreas de agrotóxicos utilizando adjuvantes. Engenharia Agrícola, v.13, p.130-135, 2005.

Cunha, J. P. A. R.; Moura, E. A. C.; Silva Junior, J. L. ; Zago, F.; Juliatti, F. Efeito de pontas de pulverização no controle químico da ferrugem da soja. Engenharia Agrícola, v.28, p.283-291, 2008.

Cunha, J. P. A. R.; Teixeira, M. M. Características técnicas de bicos de pulverização hidráulicos de jato plano Revista Brasileira de Engenharia Agrícola e Ambiental, v.5, p.334348, 2001.

Cunha, J. P. A. R.; Teixeira, M. M.; Vieira, R. F.; Fernandes, H. C.. Deposição e deriva de calda fungicida aplicada em feijoeiro, em função de bico de pulverização e de volume de calda. Revista Brasileira de Engenharia Agrícola e Ambiental, v.9, p.133-138, 2005.

Cunha, J. P. A. R.; Teixeira M. M.; Vieira, R. F.; Fernandes, H. C.; Coury, J. R.. Espectro de gotas de bicos de pulverização hidráulicos de jato plano e de jato cônico vazio. Pesquisa Agropecuária Brasileira, v.39, p.977-985, 2004.

Dallagnol, L. J.; Navarini, L.; Balardin, R. S.; Gosenheimer, A.; Maffini, A. A. Dano das doenças foliares na cultura do arroz irrigado e eficiência de controle dos fungicidas. Revista Brasileira de Agrociência, v.12, p.313-318, 2006.

Ellis, M. C. B.; Tuck, C. R.; Miller, P. C. H. The effect of same adjuvants on sprays produced by agricultural flat fan nozzles. Crop Protection, v.16, p. 41-50, 1997.

Hislop, F. C. Electrostatic ground-rig spraying: An overview. Weed Technology, v.2, p.94-105, 1988.

Monteiro, M. V. M. Compêndio de aviação agrícola. 2.ed. Sorocaba: Cidade, 2007. 298p.

Murphy, S. D.; Miller, P. C. H; Parkin, C. S. The effect of boom section and nozzle configuration on the risk of spray drift. Journal of Agricultural Engineering Research, v.75, p.127137,2000
Oliveira, J. R. G.; Ferreira, M. C.; Román, R. A. A. Diferentes diâmetros de gotas e equipamentos para aplicação de Inseticida no controle de Pseudoplusia includens. Engenharia Agrícola, v.30, p.92-99, 2010.

Ozeki, Y.; Kunz, R. P. Manual de aplicação aérea. São Paulo: Ciba Agro., 1989. 45p.

Román, R. A. A.; Cortez, J. W.; Ferreira, M. C.; Oliveira, J. R. G. di. Cobertura da cultura da soja pela calda fungicida em função de pontas de pulverização e volumes de aplicação. Scientia Agraria, v. 10, p.223-232, 2009.

Schröder, E. P.; Loeck, A. E. Avaliação do sistema de pulverização eletrostática aérea na redução do volume de calda e dosagem do herbicida glifosate. Revista Brasileira de Agrociência, v.12, p.319-323, 2006.

Silva, O. R. R. F. da; Marques, L.; Carvalho, O. S.; Vieira, R. M. Avaliação do depósito de gotas através da pulverização eletrostática e da convencional sobre a cultura do algodoeiro. Revista Brasileira de Engenharia Agrícola Ambiental, v.1, p.101-105, 1997.

SOSBAI - Sociedade Sul-Brasileira de Arroz Irrigado. Recomendações técnicas da pesquisa para o Sul do Brasil. Santa Maria: SOSBAI, 2005. 159p.

Souza, R. T.; Castro, R. D.; Palladini, L. A. Depósito de pulverização com diferentes padrões de gotas em aplicações na cultura do algodoeiro. Engenharia Agrícola, v.27, p.75$82,2007$.

Turner, C. J.; Huntington, A. J. The use of a water sensitive dye for the detection and assessment of smal spray droplets. Journal of Agricultural Engineering Research, v.15, p.385$387,1970$.

Wolf, R. E. Strategies to reduce spray drift. Kansas: Kansas State University, 2000. 4p. Application Technology Series.

Womac, A. R.; Goodwin, J. C.; Hart, W. E. Comprehensive evaluation of droplet spectra from drift reduction nozzles. St. Joseph: ASAE, 1997. 47p. 